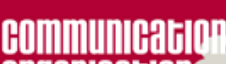

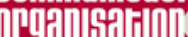

Communication et organisation

Revue scientifique francophone en Communication

organisationnelle

5 | 1994

La communication interne : une approche croisée

\title{
Communication interne et culture d'entreprise au japon
}

\author{
Renaud de Maricourt
}

\section{OpenEdition}

Journals

Édition électronique

URL : https://journals.openedition.org/communicationorganisation/1719

DOI : 10.4000/communicationorganisation. 1719

ISSN : 1775-3546

\section{Éditeur}

Presses universitaires de Bordeaux

\section{Édition imprimée}

Date de publication : 1 mai 1994

ISSN : 1168-5549

Référence électronique

Renaud de Maricourt, "Communication interne et culture d'entreprise au japon », Communication et organisation [En ligne], 5 I 1994, mis en ligne le 19 décembre 2012, consulté le 21 septembre 2021.

URL : http://journals.openedition.org/communicationorganisation/1719 ; DOI : https://doi.org/

10.4000/communicationorganisation.1719

Ce document a été généré automatiquement le 21 septembre 2021.

(C) Presses universitaires de Bordeaux 


\title{
Communication interne et culture d'entreprise au japon
}

\author{
Renaud de Maricourt
}

1 Une expression à la mode pour désigner la société moderne, au Japon, est kodojoho shakaï, ou "société de haute information"; elle englobe aussi bien la circulation interne de l'information que son acquisition. En ce qui concerne cette dernière, les manageurs nippons ont bien compris qu'elle ne sert pas à grand chose si l'information acquise n'irrigue pas aussitôt tout le corps vivant qu'est l'entreprise, si elle n'est pas convenablement partagée de façon à pouvoir être utilisée très vite, au mieux des intérêts de l'ensemble: par défaut d'organisation ou pour des raisons de pouvoir, c'est ce qui arrive souvent, pourtant, dans nos firmes. De fait, une des caractéristiques des entreprises japonaises les plus performantes est leur remarquable maîtrise des flux de communication interne. Ce phénomène est moins souvent mis en avant que les systèmes de production, de gestion des stocks ou de management des ressources humaines, par exemple, pour expliquer l'avance prise par ces sociétés, alors que pourtant, d'après les Japonais eux-mêmes, il en constitue un des ressorts. Nous essayerons ici non seulement d'analyser les modes de circulation de l'information dans les entreprises nippones, mais aussi de faire ressortir les liens entre la communication interne et la culture d'entreprise, la philosophie d'entreprise et la motivation du personnel.

\section{Culture d'entreprise}

2 Les gens à l'intérieur d'un groupe n'échangent des informations que s'ils sont animés par une volonté de partage. Une communication interne forte est d'abord un phénomène affectif; elle suppose une forte culture interne, ce qu'on appelle, dans les sociétés, «l'esprit maison » ou la « culture d'entreprise ». En même temps, elle y contribue.

3 Tout groupe humain d'une certaine stabilité tend à développer sa propre culture faite de divers signes de reconnaissance. On ne s'étonnera pas qu'au Japon, compte-tenu du système social et du paternalisme en vigueur $^{1}$, cette culture d'entreprise soit 
particulièrement forte, et qu'elle soit entretenue avec un zèle extrême. Elle se manifeste de multiples façons dans le travail quotidien, et contribue puissamment à la motivation du personnel. Elle est fondée sur des valeurs, normes et représentations communes qu'elle cherche à développer : d'abord des valeurs « défensives » selon les termes de Mike Burke², telles que la fidélité ou esprit maison, ou le culte « d'hommes-totems » (souvent, le père-fondateur) que l'on cherche à enraciner; mais elle ne néglige pas les valeurs "adaptatives» telles que le travail en équipe, la complémentarité, la tolérance, l'initiative, voire les valeurs « visionnaires » telles que l'enthousiasme, la remise en cause, l'innovation. Elle commence par l'endoctrinement systématique des jeunes, lors de sessions de formation qui constitueront la première étape de leur carrière ; jeunes que l'on aura préalablement sélectionnés selon des critères de conformité au modèle maison. Les dirigeants nippons cherchent t-ils à « maîtriser » la culture à leur avantage, à orienter les normes dans le sens de leurs intérêts, ou bien n'y a t-il pas plus simplement une mise en œuvre par eux de la culture dominante de la société japonaise dans les principes concrets devant guider l'action quotidienne de leurs collaborateurs, comme le suggère Nadine Lemaître ${ }^{3}$ ?

\section{Culture d'entreprise et communication interne}

4 Cette culture d'entreprise est d'abord une affaire de communication interne : celle-ci est très forte dans les entreprises japonaises. Turcq ${ }^{4}$ note trois principes d'organisation appliqués par elles à la communication interne, qui pourraient être de portée universelle :

5 - Multiplier les émetteurs et les circuits de communication dans l'entreprise (mieux vaut trop communiquer que pas assez, la redondance ne nuit pas),

6 - Multiplier les récepteurs (mieux vaut informer trop de gens que pas assez),

7 - Adopter des structures simples et souples facilitant la circulation de l'information, avec des comités ad-hoc constitués pour des projets spécifiques : choix d'un slogan ou d'un nom de produit, par exemple, ou organisation d'une manifestation, d'une commémoration...

8 Les moyens nouveaux de communication interne offerts par la télématique sont mis à profit pour l'application de ces principes. Ainsi, la société Recruit (qui s'était par ailleurs rendue célèbre, en 1988, par un scandale portant sur un délit d'initié, scandale qui avait fini par provoquer la chute du gouvernement Takeshita) utilise la messagerie électronique pour permettre à n'importe quel salarié de l'entreprise (elle en compte 6000 ) de dialoguer très librement avec leur PDG, M. Ida, mais aussi entre eux; tous peuvent suivre sur leur écran les échanges de messages, en application des principes de transparence et de circulation de l'information. En outre un journal interne, La mouette, diffuse largement dans le personnel non seulement des informations sur l'entreprise qui dans beaucoup d'autres seraient considérées comme indiscrètes ou confidentielles, mais aussi des opinions, impressions ou critiques de salariés sur les événements les concernant ; cela permet d'informer tout le monde sur leur véritable état d'esprit ${ }^{5}$.

\section{Culture d'entreprise et rites}

9 La culture d'entreprise est aussi faite de rites, de pratiques qui relèvent typiquement de la gestion symbolique; ce sont par excellence des mécanismes de production et de reproduction de la culture, ainsi que d'acculturation des nouveaux venus. Sapir ${ }^{6}$, à ce sujet, oppose le «symbolisme de référence », procédé économique de référence comme 
l'écriture, à ce qu'il appelle le «symbolisme de condensation »: soit «une forme très ramassée de conduite substitutive qui permet de libérer instantanément une tension affective sous forme consciente ou inconsciente ». Il remarque que les deux vont souvent de pair : des symboles de référence comme les badges, slogans, certaines façons de parler ou certains sigles « prennent facilement l'allure de rites affectifs et revêtent soudain pour l'individu et pour la société une importance considérable en tant que formes substitutives de l'expression affective ».

Dans le contexte de l'entreprise, certains de ces rites ont un caractère intégrateur, ils visent à renforcer la cohésion du groupe, comme les cérémonies initiatiques d'accueil des nouveaux employés; d'autres ont un caractère différentiateur, ils symbolisent les barrières, les conflits tolérés ou les différences souhaitées, comme les marques de la hiérarchie, les compétitions entre équipes ou services; d'autres encore marquent les frontières de l'organisation, ce sont les processus d'exclusion, les sanctions vis-à-vis des déviants, renforçant les valeurs du groupe dominant. Le monde du travail au Japon, héritier en cela de la tradition confucéenne qui donne une place très importante aux rites, offre un large éventail de toutes ces pratiques : citons-en ici quelques exemples.

\section{Réunions matinales d'accueil (" chorei shiki »)}

11 On y donne les consignes particulières pour la journée et le kacho distribue les tâches; cette fonction simple d'organisation s'enrobera volontiers d'un discours moralisateur où, à grand renfort de principes éthiques ("qualité», "service du client», "paix», "prospérité », "progrès ", " amélioration »), l'on essaye de galvaniser les énergies en rappelant des records antérieurs à battre, un autre service qu'il faut dépasser, un concurrent qu'il faut vaincre. Rituels tribaux pour nous, ces réunions sont bien acceptées du personnel qui y voit une manifestation de l'intérêt que lui portent ses supérieurs et qui joue volontiers le jeu.

\section{Hymne d'entreprise («Shaka »)}

12 Il est chanté quotidiennement dans les ateliers de certaines entreprises, seulement parfois dans d'autres, ou, dans d'autres encore, déversé par haut-parleur tous les matins. Mais tous doivent le connaître et l'entonner à l'occasion comme signe de ralliement, par exemple lors de la fête de nouvel an ou après une beuverie en groupe dans un bar. Ces hymnes ont en général un fort pouvoir fédérateur par leur rythme entraînant et par leurs paroles au sens à la fois suffisamment vague et général pour que tous y acquiescent, et en même temps à fort contenu émotionnel. Voici, par exemple, les paroles de l'hymne de la société Matsushita ${ }^{7}$ :

«Pour édifier le nouveau japon Durcissez vos efforts

Faisons le maximum pour développer notre production

Envoyons nos produits aux peuples du monde

Sans relâche, sans relâche

jaillis, industrie, jaillis,

Harmonie et sincérité est notre devise,

Celle de Matsushita Electric ». 


\section{Devise et slogans} tenues de travail uniformes dans les ateliers, et parfois aussi par certains personnels des bureaux (surtout féminins) ; par les emblèmes traditionnels d'entreprise (noren) ou par les logos plus modernes arborés par tous sous forme de badges ou d'insignes (shasho); enfin par les drapeaux d'entreprise flottant fièrement devant chaque établissement de la firme. Tous ces éléments contribuent à renforcer le sentiment d'appartenance au groupe. Certains observateurs étrangers en sourient ou se posent des questions sur leur efficacité tout en notant leur côté infantilisant. Mais ce qu'on pourrait appeler le patriotisme de société et le sens de solidarité, de cohésion, d'harmonie cultivés par ces moyens concourent effectivement à la motivation du personnel, comme le montrent diverses études faites à ce sujet $»^{10}$.

\section{Gymnastique de groupe}

Cette pratique caractéristique dans les ateliers - une pause de quelques minutes deux ou trois fois par jour (en général avant le travail, pendant la matinée ou en milieu d'aprèsmidi) au cours de laquelle les ouvriers sont invités par haut-parleur à s'adonner à divers exercices d'assouplissement scandés musicalement - peut passer à première vue pour une simple mesure d'hygiène physique et mentale. Son caractère collectif en fait aussi un moyen d'entraînement à la discipline de groupe, et un des outils de cette culture fusionnelle systématiquement recherchée dans le modèle nippon.

Concluons ces observations sur la culture d'entreprise au Japon en notant qu'à un certain reflux, voire à la contestation ouverte de celle-ci dans les années 80 succède depuis peu, avec la montée de la crise des années 91-92, un retour en force de sa popularité, comme 
en témoignent fin 1992 diverses émissions de TV, publications et bandes dessinées populaires. Ainsi une comédie musicale de Katsuhiko Ishizuka intitulée significativement "La médaille d'or du salarié » et qui fait courir tout Tokyo remet à la mode les "employés modèles ", objets de satire auparavant. L'hymne d'entreprise chanté à la fin, « les fans de l'entreprise ", a obtenu un succès triomphal. Selon un journaliste du Nikkei Ryutsu Shimbun de Tokyo $"^{11}$, "Les jeunes, particulièrement sensibles aux tendances de l'époque... se ruent aux spectacles et dévorent les bandes dessinées qui prônent le dévouement à l'entreprise et l'ardeur au travail. La rapidité du changement est étonnante ».

Il ne faut certes pas trop prendre au sérieux ces flux et reflux de la mode qui, comme des vagues à la surface de l'océan, agitent le paysage social. Force est cependant de reconnaitre que le courant de fond vers une normalisation du Japon ne progresse qu'avec une grande lenteur et bien des méandres. Que ce soit en matière de culture d'entreprise, de durée du travail ou de mode d'exercice du pouvoir, les traditions restent tenaces, derrière le paravent des mots d'ordre et les slogans.

\section{Philosophie d'entreprise et mission}

Les Japonais, « animaux stratégiques » suivant l'expression de Turcq ${ }^{12}$, aiment se projeter dans l'avenir et élaborer des plans. Et le préambule du plan d'entreprise, dans lequel sont explicitées en termes généraux la philosophie de l'entreprise et sa mission (ou projet), en constitue, pour eux, une partie importante. Des extraits choisis de ce préambule seront volontiers lus et commentés lors des réunions matinales du personnel; on y fera à l'occasion référence lors de séances de travail de cercles de qualité ou de toutes sortes d'autres comités et instances. L'essentiel en est constitué le plus souvent par des déclarations qui seraient qualifiées par les Français de bonnes intentions vagues et pompeuses. Voici, par exemple, un préambule de plan stratégique de 1987 d'une importante société de matériel électronique, Toshiba ${ }^{13}$.

Toshiba contribue à une vie plus riche et plus saine, et à l'avancement de la société par la création de nouvelles valeurs basées sur le respect de l'Homme. En particulier Toshiba :

1. Est orienté vers les consommateurs (clients) et suit une politique de priorité au consommateur,

2. Veut poursuivre ses activités en tant qu'entreprise mondiale et les mener avec une vision internationale,

3. Met l'accent, dans ses opérations mondiales, sur la coopération et sur des activités utiles à la communauté,

4. Donne à son personnel la possibilité de pleinement se réaliser et de développer au maximum ses capacités,

5. Utilise efficacement ses ressources,

6. Travaille en harmonie avec la nature et cherche à éliminer toutes les formes de pollution,

27 7. S'efforce de faire des bénéfices raisonnables, d'une façon qui soit profitable à ses actionnaires, à ses employés et à la société.

En fait, le flou des mots dissimule des intentions relativement précises : accent mis sur le développement international plutôt que sur le marché intérieur, stratégie de joint- 
ventures (« coopération »), introduction du concept écologique nouveau à cette époque pour cette société.

Autres exemples ${ }^{14}$, la société Shiseido (produits de beauté et cosmétiques) annonce dans son rapport annuel 1991 son « Grand Dessein » qu'elle résume en un slogan : «L'art de la beauté à travers la science ", et qu'elle détaille en quelques priorités :

- Aider les personnes, partout dans le monde, à exprimer leur véritable beauté intérieure et extérieure ;

- contribuer au bien-être émotionnel et physique ;

32 - procurer une palette variée de produits de qualité ;

33 - jouer un rôle actif dans la vie culturelle et sociale des consommateurs ;

34 - poursuivre une longue tradition de capacités technologiques supérieures pour explorer de nouvelles frontières scientifiques et créer une nouvelle valeur.

De même, Canon énonce ainsi sa " vision » :

«Nous sommes convaincus que l'entreprise privée remplit un rôle supérieur qui transcende celui de simplement faire de l'argent. Dans le monde actuel morcelé par de multiples frontières - politiques, économiques, religieuses, culturelles...-, l'entreprise privée doit occuper un créneau bien particulier. Elle peut aller au-delà de ces divisions, fournir des perspectives sur les problèmes, et rapprocher les hommes. C'est pourquoi la mission d'entreprise de Canon consiste à contribuer à un monde meilleur. Nous sommes convaincus que le monde ne fait qu'un, et qu'aucune nation ne peut réellement réussir aux dépens d'une autre. Nous nous engageons à conduire nos affaires dans cet esprit, et à encourager une perspective globale partout où cela est possible.»

D'une façon générale, ces philosophies ou visions d'entreprise résistent mal à une analyse rationnelle. Après avoir connu une brève vogue en France, elles y sont maintenant souvent tournées en dérision : les visionnaires, assimilés à des mages ou à des gourous, ne sont guère appréciés de nos esprits cartésiens. Ces visions jouent pourtant un rôle fort utile : dans les entreprises où l'horizon a tendance à se rétrécir sous la pression du quotidien, elles contribuent à donner une perspective plus ample, un recul, un élan tourné vers l'avenir. Dans le monde desséché des affaires, elles apportent une note de fraîcheur et de générosité. Elément de communication interne, laquelle, pour les Japonais, ne fait qu'un avec la communication externe comme nous le verrons, elles galvanisent les troupes et les aident à se tourner vers l'extérieur, à combattre la tendance naturelle - mais fatale - à l'introversion que l'on constate dans tous les groupes. En cela, elles contribuent à imprimer cet esprit marketing si nécessaire dans une économie de marché, et beaucoup plus présent dans les entreprises nippones que dans les nôtres.

Une analyse sémiologique révélera mieux la portée de ces documents : ce sont souvent des mots mis bout à bout sans signification précise, mais où chaque terme contribue sémantiquement à la formation d'une impression générale. Ils sont souvent repris en abrégé, sous forme de slogan publicitaire et de signature de la marque, comme nous l'avons vu pour Shiseido. Citons-en quelques autres exemples; pour la bière Asahi : «Live Asahi for Vive people»; ou pour les automobiles Nissan : «Feel the beat »; ou encore pour les produits de beauté Kanebo : "For beautiful human life » (ces slogans sont utilisés tels quels en anglais, au Japon). 


\section{Coordination horizontale}

Aoki ${ }^{15}$ pour expliquer le processus de prise de décision dans les firmes japonaises, propose de sortir des schémas habituels opposant pouvoir ascendant et pouvoir descendant. L'entreprise japonaise-type, d'après lui, fonctionne suivant un modèle dit de « coordination horizontale ». Le baptisant «modèle J », il l'oppose au modèle occidental traditionnel de coordination hiérarchique qu'il appelle «modèle $\mathrm{H}$ ». Le modèle J est fondé sur le partage de connaissances et l'échange d'informations permanents à la base entre différentes unités ou services, d'où des effets de rétroaction et d'ajustement mutuels spontanés. Les échanges d'information se font surtout de façon informelle et orale: d'où un gain en rapidité de prise de décision, et la possibilité d'exploiter des informations trop intuitives pour être saisies utilement sous forme écrite. La rotation systématique des postes est un des principaux moyens utilisés pour éviter les cloisonnements : les ingénieurs, par exemple, alternent les fonctions techniques et de production, ce qui coupe court à la petite guerre classique entre les deux. Pour les ouvriers et employés de base, cette rotation, associée à un bon niveau d'instruction et à une formation adéquate, leur permet d'avoir une compréhension globale du processus et même une vision d'ensemble des objectifs de l'entreprise, indispensable au bon fonctionnement de cette coordination horizontale ${ }^{16}$. Sugita fait justement remarquer que le développement des activités de petits groupes - dont certaines célèbres comme les Cercles de Qualité - observé dans d'innombrables ouvrages et articles, a aussi certainement contribué à faciliter cet ajustement informel à la base ${ }^{17}$.

La hiérarchie, dans ce système, a d'une part une fonction de planification (notamment, fixation des objectifs) et d'autre part encore de contrôle, bien que celui-ci se fasse aussi, et surtout peut-être, par les pairs. Mais son existence (ou du moins son importance en nombre) se justifie surtout comme moyen de stimulation: chaque jeune ouvrier ou employé "a dans sa giberne son bâton de maréchal », pour reprendre l'expression de Napoléon concernant ses soldats. De fait, la mobilité ascendante est plus grande au Japon qu'en Europe et la stratification en castes à peu près imperméables y est bien moindre : sur les huit patrons de grandes entreprises japonaises dont la carrière est décrite par Kamioka ${ }^{18}$, quatre ont commencé comme simples apprentis-ouvriers avec un diplôme de l'enseignement primaire ou secondaire pour tout bagage. La perspective de pouvoir gravir les échelons de la hiérarchie stimule donc le zèle de chacun et Aoki établit ainsi un des principes de son "modèle $\mathrm{J}$ » $^{19}$ : «Afin que les entreprises constituent des organisations efficaces et à forte cohésion interne, il faut que soit leur mode de coordination, soit leur mode de stimulation inclue une forte dimension hiérarchique. Comparées à d'autres, les firmes japonaises tendent à donner moins d'importance à la hiérarchie dans la gestion de la coordination, tandis qu'elles fondent leur système de stimulation sur la hiérarchie des grades.»

41 Aoki note aussi que la coordination hiérarchique, la spécialisation et la stimulation par le salaire au rendement, qui caractérisent le modèle taylorien encore assez largement en vigueur en Occident, fonctionnent probablement mieux soit dans un contexte stable (où l'autorégulation à la base et l'ajustement des directives et plans de la hiérarchie ne sont guère nécessaires), soit, au contraire, dans un environnement très instable, de crise (où la base a tendance à sur-réagir et où la régulation par le haut redevient nécessaire, car on y a plus de recul par rapport à l'événement immédiat). C'est dans les situations 
intermédiaires, lorsque l'environnement change constamment mais pas trop fortement, que le « modèle J » s'avère supérieur; il se trouve aussi que ce sont les situations les plus fréquentes dans l'industrie aujourd'hui.

Il se pourrait que cette coordination horizontale informelle fondée sur l'élimination des barrières à la communication interne soit une des clés cachée des nouveaux modèles de production à la japonaise, du type « juste à temps » ou " qualité totale », souvent imités en Occident mais sans que leurs fondements soient toujours bien compris, d'où de fréquentes déceptions ${ }^{20}$.

\section{Circulation de l'information, communication interne et organisation personnelle}

Tous les éléments du management des ressources humaines au Japon concourent à assurer une circulation interne de l'information aussi rapide et aussi intense que possible; la coordination horizontale dont nous venons de parler ne peut fonctionner bien qu'à cette condition. Les responsables ont bien compris que l'information était la clé de l'efficacité. Tout est donc mis en œuvre pour faciliter cette circulation, tant horizontale que verticale, ascendante que descendante : panneaux d'affichage, journaux d'entreprise, moyens techniques modernes (réseaux d'ordinateurs, fax, réseaux vidéo internes...), moyens psychologiques pour éviter les phénomènes de rétention d'information, systèmes d'organisation en équipes communicantes, échanges de personnel. L'information doit franchir les barrières hiérarchiques, de fonctions, de services.

Même la disposition des bureaux favorise les échanges d'information informels : grands bureaux collectifs où chacun voit ce que font les autres, les entend, peut les interpeller ou leur transmettre immédiatement un document; le chef de service (kacho) est au milieu de ses employés ou plus souvent leur fait face (cf. graphes 1 et 2). Cette disposition des bureaux qui, notons-le, était usuelle en Occident avant la deuxième guerre mondiale et réapparaît timidement avec la mode des "bureaux paysages ", constitue une pièce non négligeable du système de management des ressources humaines au Japon. Il existe une hiérarchie dans les emplacements: les employés les plus importants (plus anciens ou mieux en cours) prennent les bureaux les plus proches du chef, juste en face de lui et au milieu, les jeunes se situent sur les côtés, plus loin et plus en arrière, et les vieux sur une voie de garage (les «mangeurs de riz froid " selon une expression japonaise imagée) se voient affecter les coins les plus éloignés. Certains bureaux contiennent une centaine, voire plusieurs centaines d'employés: dans ce cas, chaque service dispose d'un «territoire " qui peut être partiellement délimité par des étagères, des placards ou des cloisons à mi-hauteur. Les bucho disposent souvent d'un espace semi-privé de quelques mètres carrés, sorte de cage vitrée d'où ils ont vue sur leurs subordonnés. La persistence au Japon de cette topologie des bureaux en grands espaces ouverts où travaillent plusieurs dizaines d'employés symbolise bien la force de la culture tribale dans ce pays.

Graphique 1 - Plan-type d'une salle de bureaux administratifs ou commerciaux

Source : TURCQ D. L'animal stratégique - l'ambiguiité du pouvoir chez les cadres japonais (Ed.de I'EHESS, 1985), p. 174. 


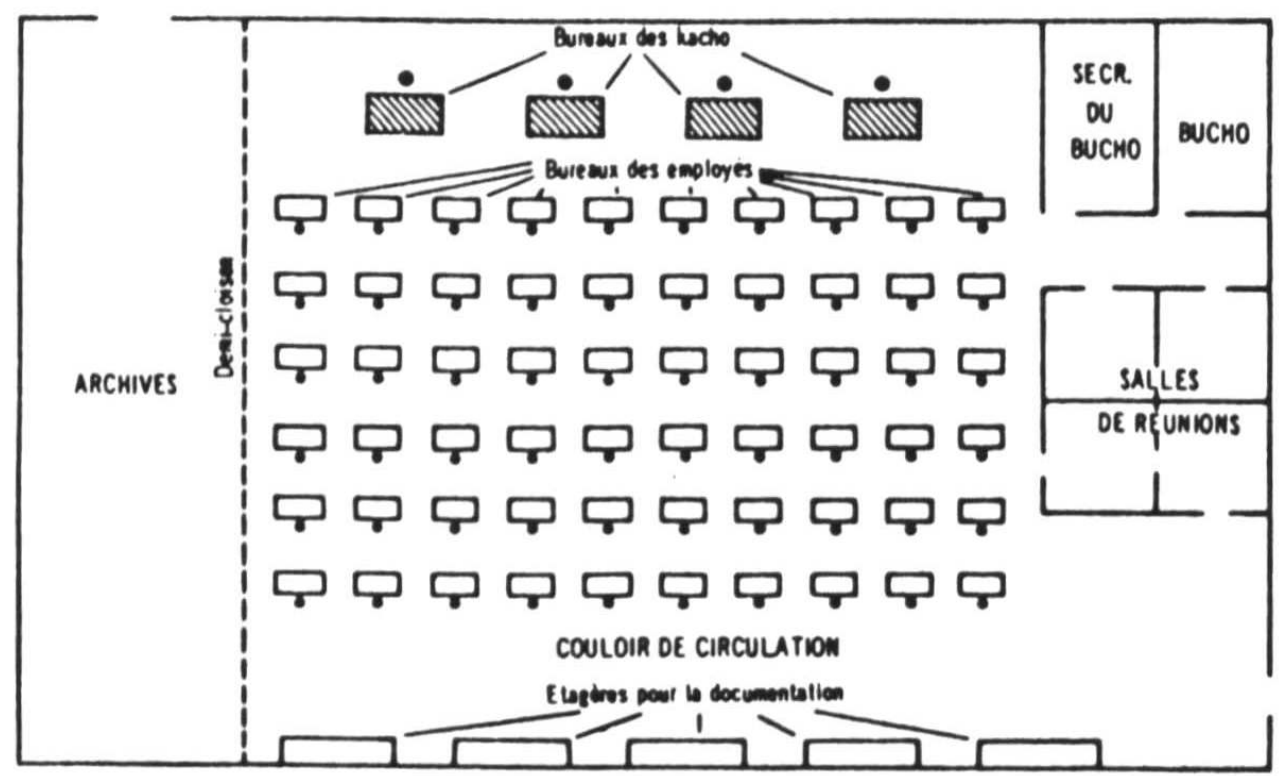

Graphique 2 - Plan-type de bureaux d'un service technique

Source : TURCQ D. op. cit. p. 175.

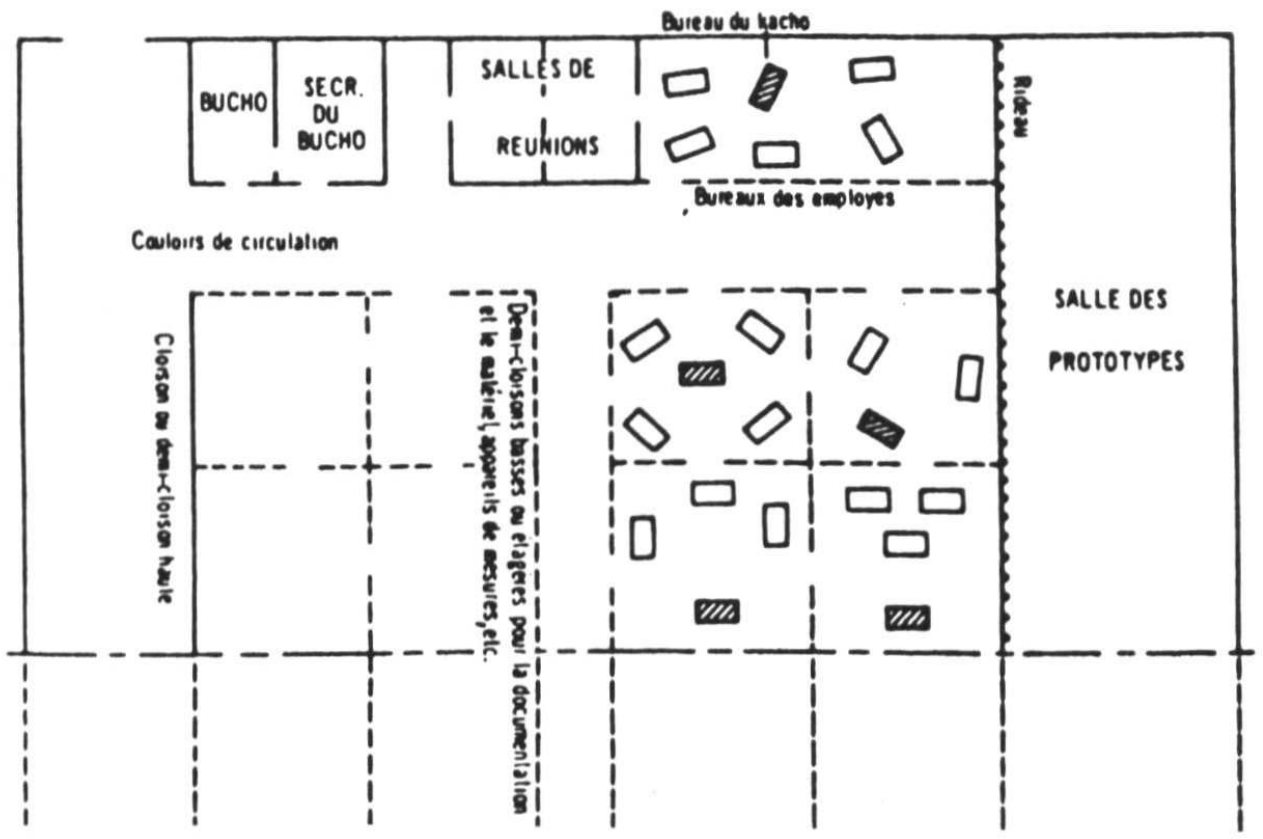

Turcq ${ }^{21}$ analyse ainsi l'efficacité de cette configuration en termes de communication interne et de circulation de l'information :

«Les subordonnés sont alignés comme à la revue ou comme dans les écoles, face au bureau du chef de section... Chacun, au milieu du bruit (plutôt du brouhaha), par-dessus ses tâches, entend les conversations de ses voisins, est informé de toutes les actions en cours dans sa section et restitue en permanence ses propres actions dans cet ensemble... L'ensemble de l'entreprise est une sorte de caisse de résonance. Chacun, par le journal interne, les feuilles d'information, l'information hiérarchique écrite ou orale, se tient à l'écoute. Chacun, collant son oreille au sol, entend vivre les environs et adapte son 
attitude. Assurer une grande diffusion de l'information générale et sensibiliser chacun à la prendre en considération ne signifie pas que chacun doive se mêler de tout mais qu'au contraire l'information peut (doit?) intéresser tout employé, même marginalement, et doit lui permettre d'ajuster son action. En d'autres termes, l'information générale n'est pas un outil de pouvoir, c'est un outil de travail.»

Seuls les grands directeurs ont droit à un bureau particulier, et encore pas toujours. Whitehill ${ }^{22}$ cite le président de Honda America Motors (filiale de Honda employant plus de 5000 personnes aux États-Unis) qui, interrogé par un journaliste sur les raisons pour lesquelles il n'avait pas droit à son propre bureau, lui répondit : «J'ai un espace de travail spacieux que je partage avec d'autres employés. Quand nous travaillons ensemble dans cette grande salle, nous pouvons nous parler tranquillement les uns aux autres. Nous échangeons des tas d'idées et de suggestions, dans ces conversations ».

Les très fréquentes réunions, les comités de toutes sortes sont encore des moyens d'échange d'information: la société de haute information est une société de haute réunionite. Les pauses aussi contribuent à la discussion, et l'expression familière «chotto ippai'(littéralement : « un peu soif», soit : «allons boire un pot ») est une invite courante à aborder de façon détendue un problème. À nouveau le soir après le travail, plusieurs fois par semaine, l'équipe se retrouve dans un bar avec son chef pour papoter sur les événements de la journée (manière comme une autre de se mettre mutuellement au courant) et pour discuter des problèmes du service avec la franchise que permet l'alcool : sous prétexte d'être un peu ivre, on peut se dire des choses que l'on n'oserait pas exprimer autrement. On fera semblant d'avoir tout oublié le lendemain, mais le message sera passé; c'est là un moyen de régulation classique dans les sociétés japonaises, informel mais efficace.

Un autre aspect du fonctionnement des entreprises japonaises qui frappe les observateurs occidentaux est le mode de communication interne: dans nos cultures qui privilégient l'écrit celle-ci se fait beaucoup sous forme de notes, mémos, rapports et comptes-rendus couchés sur le papier ; alors qu'au Japon on préfère infiniment la communication orale, plus souple, plus rapide, plus chaleureuse et plus efficace, car permettant le dialogue; ou, mieux encore, la communication non verbale lorsqu'elle est possible. Des stagiaires japonais dans une entreprise française sont stupéfaits de voir des cadres occupant des bureaux voisins perdre du temps à échanger des notes qu'il leur faut rédiger, faire taper (souvent encore) par une secrétaire, corriger, enregistrer et classer, alors qu'il serait tellement plus simple de se lever de son bureau ou de soulever son combiné téléphonique et de parler ensemble. On a parfois prétendu qu'on ne s'écrit pas beaucoup dans les entreprises japonaises à cause de la difficulté de taper à la machine les kanjis (idéogrammes). Cette difficulté a pratiquement disparu, maintenant, avec les ordinateurs et les logiciels de traitement de texte. En fait la raison est plus profonde, elle est d'ordre culturel. Dans notre rationalité, la parole est frivole, elle n'engage qu'à moitié et elle ne dure pas : «verba volent » (les paroles s'envolent) dit un adage latin; l'écrit rassure, lui, il est précis, définitif. La culture japonaise est fondée sur la notion d'impermanence des choses et, pour reprendre la métaphore de la fable de La Fontaine, elle préfère au chêne le roseau : la flexibilité de l'un plutôt que la solidité (ou la rigidité) de l'autre, et la parole ou mieux encore la non-parole, l'entente à demi-mot fondée sur la connivence - plutôt que le papier. Seules les communications importantes, comme dans la procédure formalisée du ringi, se font par écrit - et encore, le plus brièvement possible. 

secrétaire. Cette culture du silence en est la cause, et les analystes estiment que les entreprises y gagnent en efficacité. Chacun tape lui-même son courrier et classe ses dossiers. On fait peu de rapports écrits, et ceux-ci, lorsqu'il y en a, sont brefs et factuels, suivant souvent un plan et un format standardisés pour gagner du temps. Chez Suntory (numéro un des whiskys et spiritueux), par exemple, les plans de marketing pour le lancement de nouveaux produits, tout en donnant les renseignements essentiels, tiennent en une seule page. Le volume de communication écrite étant, dans les sociétés nippones, au moins trois à quatre fois moins important que chez nous, on y passe moins de temps en recherche de documents, en rangements et en classements. L'organisation à la japonaise, c'est aussi, ne l'oublions pas, le «zéro papier ».

51 Dans la tradition zen, la sobriété est de mise : on écrit peu, mais on parle aussi peu. « $\mathrm{L}$ »homme véritable se tait et boit de la bière Sapporo » disait une publicité célèbre des années $70^{23}$. La « culture de l'oral » japonaise est plutôt une culture du non-écrit et de la connivence (an mokuchi): on y prise par-dessus tout l'art du silence, contrairement à la France, pays de la rhétorique. Avec les « gens du dedans » les mots, qu'ils soient écrits ou énoncés oralement, sont superflus; on recherche plutôt la compréhension de l'autre par l'empathie, acquise à la suite d'une longue fréquentation mutuelle. D'où l'importance du temps, avec l'emploi à vie, dans le mode japonais de gestion des ressources humaines.

On retrouve cette sobriété dans la décoration ou l'équipement, dans les ateliers et les bureaux. Ainsi, contrairement à ce qu'on pourrait croire, l'équipement bureautique est souvent assez limité, dans les grandes sociétés, ce qui oblige à partager et favorise, une fois de plus, l'esprit de groupe. Une étudiante française en stage au siège de Suntory à Tokyo pendant l'été 1993 témoigne ${ }^{24}$ :

«Les ressources informatiques sont révélatrices d'une gestion parcimonieuse et soucieuse de ne pas habituer l'employé Suntory au superflu. Pour une unité... employant une cinquantaine de personnes, il n'y avait pas plus de quatre à cinq ordinateurs; ce léger équipement informatique était complété par des sortes de petits ordinateurs conçus pour le seul traitement de texte, portables, que les personnes allaient chercher dans un coin de la pièce quand elles en avaient besoin. Il est vrai que les ordinateurs de bureau sont largement utilisés à des fins de traitement de texte et peuvent alors être avantageusement remplacés par des machines plus simples de moindre coût, les ordinateurs étant réservés à l'utilisation de tableurs, bases de données ou logiciels de dessin. Par ailleurs, la relative limitation des moyens informatiques impose un partage de ces moyens et évite donc l'inflation de temps passé devant les ordinateurs pour traiter les chiffres à un niveau de détail trop fin pour être opérationnel ou encore rendre beau un schéma dont on a simplement besoin qu'il soit clair. Ce partage imposé des ressources impose donc de travailler efficacement et de n'employer l'ordinateur qu'à des fins utiles, idée intéressante à l'heure où des études américaines montrent que le développement de l'informatique n'a pas accru la productivité des cols blancs, tentés devant ces machines de déployer un perfectionnisme inutile.»

'on économise l'équipement on s'adonne volontiers, en revanche, aux réunions, entretiens ou discussions, formelles ou informelles, bilatérales ou multilatérales: les Japonais, contrairement à nous, n'y voient pas une perte de temps ; ils pensent qu'on se comprend mieux en parlant (même à demi-mot), et que les notes sont une source de malentendus et de conflits, dans la mesure où elles figent trop une pensée ou une situation. Autre avantage dans un pays où l'espace manque, on y gagne de la place.

Communication et organisation, 5 | 2012 
Chacun n'a qu'un bureau de dimension plus restreinte que chez nous, et il serait impossible d'y stocker des dossiers volumineux, incitation supplémentaire à se parler, à écouter, à deviner surtout, et à ne pas s'encombrer de paperasses.

Quelques signes donnent toutefois à penser que certains excès de ce système hypercommuniquant commencent à être remis en cause. Dans un contexte de restrictions, bien des sociétés s'aperçoivent que leur budget de communication s'est démesurément gonflé avec le temps. Ainsi, un responsable des affaires administratives de la société de commerce Sumitomo a été effaré de constater, en 1993, que les 6750 employés de la firme consommaient quotidiennement deux millions de feuilles de papier en photocopies et reproductions diverses, et qu'ils échangeaient 30000 à $40000 \mathrm{fax}$ ou telex avec les multiples filiales et sociétés-sœurs ou les 130 bureaux à l'étranger. De même, on commence à prendre conscience que les multiples réunions finissent par coûter fort cher en temps. Un responsable de la comptabilité d'Hitachi a calculé le coût des réunions passées à la préparation des budgets semestriels par les quelque 28000 cadres et employés de la société qui y consacrent la moitié de leur temps pendant deux mois par an ; il est arrivé au chiffre colossal de 17 milliards de yens, soit plus de 800 millions de F. : $30 \%$ du bénéfice d'exploitation escompté pour $1992-1993^{25}$. Aussi, depuis la crise, certains patrons, adeptes du re-engineering, tentent de limiter les excès en la matière: chez Mitsubishi Electric, par exemple, la durée des réunions a été limitée à une heure ${ }^{26}$.

Se pose donc la question de savoir si les coûts de la tant vantée gestion consensuelle n'excèdent pas ses bénéfices lorsqu'elle est poussée trop loin, et si trop de communication ne finit pas par tuer la communication; la tendance est alors de revenir à des pratiques plus sages, et plus proches, finalement, de celles des sociétés occidentales.

\section{Suggestions}

57 On peut considérer celles-ci comme un cas particulier de circulation de l'information, qu'il est intéressant d'analyser de façon plus approfondie en raison de l'importance qu'elles revêtent aux yeux des manageurs japonais et de la contribution des systèmes de suggestions à leurs performances.

L'idée de mettre en place de tels systèmes allant au-delà de la simple boîte à idées passive vient des États-Unis. Elle fut importée dans les entreprises Japonaises après la seconde guerre mondiale, et progressivement améliorée et adaptée à leur culture particulière. Les Américains, bien dans l'esprit taylorien, raisonnaient sur le triple principe de suggestions individuelles, de motivation financière pour celui qui émet la suggestion, et d'avantage économique pour l'entreprise; les Japonais, tout en encourageant la suggestion individuelle, mirent davantage l'accent sur la suggestion de groupe d'une part (avec par exemple les cercles de qualité), sur les motivations et les avantages psychologiques d'autre part. Aujourd'hui, d'après Imai ${ }^{27}$, presque toutes les grandes sociétés et la moitié des PME ont mis en place des systèmes de suggestions. Le nombre et la qualité des suggestions émises par les employés ou les ouvriers est un critère important d'appréciation de leurs performances : elles sont le signe qu'ils possèdent des aptitudes supérieures à celles que leur emploi requiert. Le nombre de suggestions de chaque équipe, atelier ou service est affiché, des concours sont organisés ; le plus grand soin est apporté ensuite à l'examen des suggestions, à leur discussion et amélioration éventuelle, enfin à leur application. Un des principaux bénéfices de ces systèmes, outre le progrès de productivité et les innovations souvent petites mais fréquentes qu'ils permettent, est la 
motivation du personnel : l'individu ou le groupe qui a une idée est heureux de pouvoir l'exprimer et d'être écouté ; il en est fier et, si elle concerne son travail (ce qui est le cas le plus fréquent) il l'appliquera avec plaisir même si cela entraîne pour lui un effort supplémentaire, alors que si elle venait de la hiérarchie il y mettrait beaucoup moins d'empressement.

D'après l'Association Japonaise de Relations Humaines ${ }^{28}$, les principaux thèmes de suggestions sont les suivants, dans l'ordre :

\section{lancement} capacité de l'entreprise à les évaluer et à les appliquer rapidement, comme le fait remarquer un cadre d'une usine japonaise aux États-Unis ${ }^{30}$ :

78 «Un de nos plus grands problèmes est que notre système kaïzen ne s'est pas développé aussi vite que certains de nos gens. Ils ont soumis des tas d'idées, mais nous ne les appliquons pas. Nous devons apprendre à mieux utiliser cette information et à appliquer ces idées, pour les encourager à nous en soumettre. Car nous en entendons déjà certains 
nous dire : 'Dites donc, j'ai donné dix idées, et je n'en ai vu aucune être appliquée. J’ai l'impression d'être ignoré'. Et alors on voit le robinet à idées se fermer ».

\section{Communication interne et publicité}

Pour les Japonais, communication interne et communication externe ne font qu'un. La communication interne prend volontiers un caractère nettement publicitaire, elle utilise ses médias et son langage. Elle a pour caractéristique de s'articuler étroitement avec la communication "externe", c'est-à-dire par exemple avec la publicité vis-à-vis des acheteurs potentiels. Mieux, cette publicité est aussi un moyen de communication interne : la communication institutionnelle de type publicitaire cherche non seulement à convaincre les publics externes de l'entreprise mais elle est aussi destinée à entretenir le moral des salariés. La cible des campagnes est donc large et dépasse de beaucoup les clients potentiels, elle inclut tous les partenaires de l'entreprise; au moins autant que le public, elle informe et motive le personnel dont il s'agit de renforcer la fierté, la cohésion, l'ardeur au travail et la conscience professionnelle. Suggère-t-on dans une publicité la qualité des produits, ou celle du service? Le but est certes de convaincre les consommateurs, mais aussi d'inciter le personnel à faire des efforts pour conformer la réalité à l'image présentée ; l'idéal tire le réel, en quelque sorte. De même les slogans sont souvent au moins autant à usage interne qu'externe, ils ont alors pour but de galvaniser les troupes. Certaines firmes françaises l'ont compris: Peugeot par exemple avec son slogan «Le lion sort ses griffes » s'adressait plus à son propre personnel qu'à des acheteurs potentiels.

Dans l'autre sens, la communication interne prépare celle tournée vers l'extérieur, elle est un gage de sa qualité et contribue à la rendre plus efficace: une bonne ambiance interne renforce la bonne image externe. Les manageurs japonais croient à ce que Turcq appelle «la continuité sociale de la communication $»^{31}:$ l'homme est un, qu'il soit employé ou consommateur; de même la communication doit être une, obéir aux mêmes principes de séduction, de motivation ou d'information, qu'elle soit interne ou externe, et passer par les mêmes canaux. Ainsi la communication interne, outre ses propres moyens d'expression (réunions, notes internes, panneaux d'affichage, par exemple) utilise de plus en plus des médias qui la rapprochent de la communication externe: T.V., avec le développement rapide, dans les grandes sociétés, des émissions de télévision d'entreprise ${ }^{32}$ et des moyens journalistiques (journaux d'entreprise). Ces derniers sont un exemple typique de la symbiose voulue entre communication interne et externe. Nombre de sociétés, en effet (un tiers des 650 plus grosses entreprises, selon Szigeti ${ }^{33}$ ) éditent des magazines souvent luxueux, à dominante culturelle et récréative, destinés autant au public qu'à leur personnel. Certaines le vendent dans leur réseau de distribution, concurrençant les maisons d'édition traditionnelles. Toujours selon Szigeti, elles veulent ainsi « participer au vaste mouvement culturel, améliorer l'image de la société, attirer de la main d'œuvre diplômée qui n'aurait pas réussi à intégrer une maison d'édition à la sortie de l'université, en lui faisant miroiter un poste au sein d'une équipe éditoriale, et accessoirement gagner de l'argent $»$. 


\section{BIBLIOGRAPHIE}

La manière dont la communication interne est organisée, entretenue, stimulée dans les entreprises japonaises les plus performantes est sans doute une des clés de leur efficacité. Ce modèle est-il transposable chez nous ? Sans doute, mais à condition d'en bien analyser les fondements. La tentation d'aller au plus vite, de ne retenir que les recettes, de ne regarder que les causes immédiates de phénomènes complexes (et le mode de management japonais en est certainement un) conduit presque toujours à des déconvenues, lorsque l'on tente de les reproduire. Dans notre cas, par exemple, il ne suffit pas de disséquer ce modèle d'intense communication pour en comprendre les mécanismes, car, derrière la communication, il y a la motivation de ceux qui communiquent, la volonté d'échanger. Cette motivation elle-même s'enracine dans un modèle de management des ressources humaines que nous appellerons l' humanocentrisme. Il se caractérise par un choix délibéré qui court en filigrane derrière tout ce que l'on considère comme des particularités du mode de gestion nippon : celui chez les responsables de privilégier le capital humain. Ce choix est fondé sur la prise de conscience qu'une organisation ou une entreprise, ce sont d'abord et avant tout des êtres humains qui, pour donner beaucoup au groupe doivent aussi, d'abord, recevoir beaucoup de lui ; non seulement en termes sèchement monétaires, mais aussi, surtout peut-être, en termes affectifs : il faut aimer pour donner. Ce modèle bien sûr n'est pas appliqué par toutes les entreprises japonaises, probablement par une minorité seulement ; il est perverti, dénaturé à l'occasion par certaines ; mais c'est tout de même le modèle de référence. C'est peut-être là un des ressorts mal connu de ce que l'on a coutume d'appeler le management japonais - en fait, une règle universelle de management -et un des secrets de la redoutable efficacité des quelques grandes entreprises de l'Empire du Soleil-Levant qui constituent le fer de lance de son armée économique.

Aoki Masahiko, Information, Incentives \& Bargaining Structure in the Japanese Economy, Cambridge Univ. Press, Cambridge \& New York, 1988 - Traduction française : Economie japonaise - Information, motivation et marchandage (Economica, 1991).

Aoki Masahiko, Toward an Economie Model of the Japanese Firm, Journal of Economic Littérature, Nashville, Tennessee, mars 1990, repris par Problèmes économiques n² 2025, 15 mai 1991.

Ballon Robert J., Foreign Competition in Japan -Human Resource Strategies, Routledge, Londres, 1991.

Baumont Marjorie, Rapport de stage à l'étranger de fin de deuxième année : Suntory, ESCP, déc. 1993.

Beaux Gilberte et al., La leçon japonaise, Plon, 1992.

Bourguignon Annick, Le modèle japonais de gestion Ed. La Découverte, 1992).

Breton Philippe, L'utopie de la communication, l'émergence de l'homme sans intérieur (Éd. La Découverte, 1992).

Burke Mike, A chacun son style d'entreprise (Inter-Éditions, 1987).

Chabanal Luc, Communiquer au Japon (Centre Français du Commerce Extérieur, 1991).

Chancel Claude, Nippon, Géo-économie d'une grande puissance (Eyrolles, Paris, 1990).

La concertation dans l'entreprise? Trop cher !, in Nihon Keizei Shimbun, repris dans Courrier international $n^{\circ}$ 131, 6 mai 1993.

La connaissance par la connivence, in Courrier international n 171, 10 févr. 1994. 
Coriat Benjamin, Penser à l'envers - Travail et organisation dans l'entreprise japonaise (Christian Bourgeois, 1991).

Fabra Paul, Kyosei, in Le Monde, 24 nov. 1992.

Fukuhara Yoshiharu, How the Japanese Motivate, in The Morning Sun, 9 Jan. 1993.

Garanto Annie, Existe-t-il un modèle japonais des ressources humaines?, in Cahiers français, $\mathrm{n}^{\circ} 279$, Cahier « Gestion des Ressources Humaines » sous la direction de Jean Yves CAPUL, (La Documentation Française, juil.-sept. 1993).

Hanada Masahori, L'entreprise japonaise et la gestion des ressources humaines, in Cipango, revue de 1TNALCO, févr. 1993.

Helms M., Communication : the Key to JIT Success, in Production \& Inventory Management, vol. $31 \mathrm{n}$ $\circ 2,1990$.

Imai Masaaki, Kaizen - La clé de la compétitivité japonaise (Eyrolles, Paris, 1989).

Ishinabe Hitomi, Théâtre, BD, télé : l'entreprise joue les stars, in Courrier International, nº 111, 17 déc. 1992 (repris de Nikkei Ryutsu Shimbun).

Japon, le consensus, mythes et réalités (Cercle d'études sur la société et l'économie du Japon, Economica, 1984).

- Japanese Management - Mission Mitsubishi, in The Economist, 19 Feb. 1994.

Kamioka Kazuyoshi, Les pionniers de l'industrie japonaise (Eyrolles, 1989).

Kenney Martin \& Richard Florida, Beyond Mass Production - The Japanese System \& its Transfer to the U.S. (Oxford University Press, New York, 1993).

Landier Hubert, L'entreprise polycellulaire - Pour penser l'entreprise de demain (Entreprise Moderne d'Edition, 1989).

Lemaître Nadine, La culture d'entreprise : outil de gestion ?, in Direction \& gestion, nov./déc. 1984.

Le Saget Meryem, Le manager intuitif - Une nouvelle force (Dunod, 1992).

Maricourt (de) Renaud, Au Japon la vente est l'affaire de tous, in Action Commerciale $\mathrm{N}^{\circ} 60$, novembre 1987.

Maricourt (de) Renaud et Roger Ourset, Tokyo Blues, in Science \& Vie économie, $\mathrm{N}^{\circ} 42$, septembre 1988.

Maricourt (de) Renaud, Le paternalisme japonais est-il importable ? Cahier de Recherche $\mathrm{N}^{\circ}$ 92-108, ESCP, 1992.

Maricourt (de) Renaud, Les Samouraïs du Management - Production, marketing et finance au Japon, Vuibert, 1993.

Maury René, Les patrons japonais parlent, Seuil, 1990.

Mercier Christian (coord.) Japon, stratégies industrielles et enjeux sociaux, Presses Universitaires de Lyon, Lyon, 1988.

Morita Keiko \& John C. Oliga, Groupism and Japanese Decision-Making Process, in Lin Chi-Yuan \& Junying Huang (ed.), Proceedings of the Fourth International Conference on Comparative Management, National Sun Yet-Sen Univ., Kaohsiung, Taiwan, 1991.

PONS Philippe, Le modèle japonais, in Le Monde, 3 mars 1993. 
Les recettes japonaises de la télévision d'entreprise, in Courrier international nº 101, 8 oct. 1992 (repris du Nikkei Sangyo Shimbun).

Recruit joue la carte du parler vrai, in Courrier international n 153, 7 oct. 1993 (repris du Nikkei Sangyo Shimbun).

Rehfeld John O., What Working for a Japanese Company Taught me in Harvard Business Review, nov.déc. 1990 - Trad, française : « Mon travail de patron dans une entreprise japonaise » in HarvardL’Expansion, été 1991.

Robinson Alan G. \& Dean M. SCHROEDER, Training, Continuous Improvement, \& Human Relations: The U.S. TWI Programs \& the Japanese Management Style, in California Management Review, hiver 1993.

Rouillault Henri, Le Japon - Croissance économique et relations de travail, La Documentation Française, 1983.

Sapir Edward, Le symbolisme, in Anthropologie, Éditions de Minuit, 1967.

Sugita Kurumi, in MERCIER Christian, op. cit.

Szigeti Pierre-Alain, Articles sur mesure, in France-Japon Eco, Chambre de Commerce et d'Industrie Française du Japon, $n^{\circ}$ 53, hiver 1992.

Tateisi Kazuma, Entreprise et créativité : l'exemple japonais, Masson, 1993.

Trinh Sylvaine, Il n'y a pas de modèle japonais, Ed. Odile Jacob, 1992).

Turcq Dominique, L'animal stratégique - l'ambiguïté du pouvoir chez les cadres japonais, Éd. de l'EHESS, 1985.

Turcq Dominique, Une communication branchée : la communication d'entreprise et le changement social au Japon, Cahiers D'Etudes Nº 87-66, ESCP, 1987.

Turcq Dominique, L'inévitable partenaire japonais : repères dans un dédale, Fayard, 1992.

Whitehill Arthur M., Japanese Management -Tradition and Transition. Routledge, Londres, 1991.

Yoshimori Masaru, Sources of Japanese Competitiveness, in Management japan, vol. 25, $\mathrm{N}^{\circ} 1$, Spring 1992.

Ziemba William T.\& Sandra L. SCHWARTZ, Power Japan : How \& Why the Japanese Economy Works, Pitman, Londres, 1991.

\section{NOTES}

1. Voir à ce sujet notre étude: de MARICOURT Renaud, Le paternalisme japonais est-il importable? Cahier de Recherche $\mathrm{N}^{\circ}$ 92-108, ESCP, 1992

2. BURKE Mike, A chacun son style d'entreprise, Inter-Éditions, 1987.

3. LEMAÎTRE Nadine, La culture d'entreprise: outil de gestion?, in Direction \& gestion, nov.-déc. 1984, p. 42.

4. TURCQ Dominique, Une communication branchée : la communication d'entreprise et le change ment social au lapon Cahier d'Etudes n 87-66, ESCP, 1987, pp. 26 à 33

5. Recruit joue la carte du parler vrai », in Courrier international n ${ }^{\circ} 153,7$ oct. 1993 (repris du Nikkei Sangyo Shimbun).

6. SAPIR Edward, « Le symbolisme », in Anthropologie, Édition de Minuit, 1967.

7. Cité par CHAN-CEL Claude, Nippon, Géo-économie d'une grande puissance, Eyrolles, Paris, 1990, p. 34. 
8. TATEISI Kazuma, Entreprise et créativité. l'exemple japonais, Masson, 1993, p. 109.

9. Ibid., p. 13.

10. WHITEHILL Arthur M, Japanese Management - Tradition and Transition Routledge, Londres, 1991, pp. 196-201.

11. ISHINABE Hitomi, ‘Théâtre, BD. télé : l'entreprise joue les stars ", in Courrier International, n -111,17 déc. 1992, repris de Nikkei Ryutsu Shimbun.

12. TURCQ Dominique, L'animal stratégique - l'ambiguïté du pouvoir chez les cadres japonais, Éd. de l'EHESS, 1985.

13. Cité par WHITE-HILL. Arthur M., op. cit.

14. Cités par LE SAGET Meryem, Le manager intuitif-Une nouvelle force Dunod, 1992, p. 77.

15. AOKI Masahiko. "Toward an Economie Model of the Japanese Firm », journal of Economic Littérature, Nashville, Tennessee, mars 1990 repris par Problèmes économiques n 2025 (15 mai 1991).

16. Sans qu'elle soit autant développée et érigée en système, la même idée se retrouve chez différents auteurs. Voir par exemple IMAI Masaaki, Kaizen -La dé de ja compétitivité japonaise Eyrolles, Paris, 1989, pp.117-118. Celui-ci donne à cette forme de coordination le nom de «management transfonctionnel».

17. SUGITA Kuru-mi, in MERCIER Christian (coord.) Japon, stratégies industrielles et enjeux sociaux (Presses Universitaires de Lyon, Lyon, 1988).

18. KAMIOKA Kazuyoshi, Les pionniers de l'industrie japonaise, Eyrolles, 1989).

19. AOKI Masahiko, op. cit.

20. HELMS M., "Communication: the Key to JIT Success", in Production \& Inventory Management, Vol. 31, $\mathrm{n}^{\circ} 2$.

21. TURCQ Dominique, Une communication branchée : la communication d'entreprise et le changement social au japo, Cahiers D'Études No 87-66, ESCP, 1987 p. 29.

22. WHITEHIL. Arthur M, japanese Management Tradition and Transition (Routledge, Londres, p. 119.

23. Cité dans «La connaissance par la connivence », in Courrier international n 171, 10 févr. 1994, p. 21.

24. BAUMONT Marjorie, Rapport de stage a l'étranger de tin de deuxième année : Suntory (ESCP, déc. 1993).

25. "La concertation dans l'entreprise? Trop cher!", in Nihon Keizei Shimbun, repris dans Courrier international $\mathrm{n}^{\circ}$ 131, 6 mai 1993, p. 21-26. "Japanese Management - Mission Mitsubishi », in The Economist, 19 Feb. 1994, pp. 77-78.

26. "Japanese Management - Mission Mitsubishi », in The Economist, 19 Feb. 1994, pp. 77-78.

27. IMAI Masaaki, Kaizen - La clé de la compétitivité japonaise, Eyrolles, Paris, 1989 pp. 15 et 104 à 114.

28. Masaaki, op. cit. p. 125.

29. MAURY René, les patrons japonais parlent, Le Seuil, 1990, p. 644.

30. KENNEY Martin \& Richard FLORIDA, Beyond Mass Production - The Japanese System \& its Transfer to the U.S., Ox-ford University Press, New York, 1993, p. 108.

31. TURCQ Dominique, Une communication branchée : la communication d'entreprise et le changement social au lapon, Cahiers D’Études, N87-66, ESCP, 1987, p. 46.

32. Voir «Les recettes japonaises de la télévision d'entreprise ", in Courrier international $\mathrm{n}^{\circ} 101$, 8 oct. 1992 (repris du Nikkei Sangyo Shimbun).

33. SZICET1 Pierre-Alain, "Articles sur mesure ", in France-lapon Eco, Chambre de Commerce et d'Industrie Française du Japon, n » 53, hiver 1992, p. 49. 


\section{RÉSUMÉS}

Le modèle japonais de management des entreprises est caractérisé par une communication interne extrêmement intense. Celle-ci est fondée elle-même sur une forte culture d'entreprise, stimulée par diverses pratiques rituelles. L'énoncé de la philosophie de l'entreprise ou de sa mission, souvent en préambule d'un plan d'entreprise, constitue une autre pratique fédératrice permettant de favoriser « l'esprit maison ». Le fort esprit de groupe et l'intense communication interne permettent un processus de prise de décision à la base par ajustement mutuel informel et spontané, dit " coordination horizontale ", qui, grâce à sa souplesse, se révèle plus performant que le modèle hiérarchique traditionnel, dans un environnement changeant. Divers moyens sont employés pour stimuler la communication interne et pour favoriser les échanges informels d'information. Parmi ces moyens, il faut aussi compter les systèmes de suggestions. Enfin, signalons que la publicité vis-à-vis des acheteurs potentiels, par exemple, est aussi un moyen de communication interne destiné à informer et motiver le personnel. Ce qui nous conduit à conclure qu'au Japon communication interne et communication externe sont étroitement imbriquées.

An extremely high level of internal communication characterizes the Japanese management model. This phenomenon is based on a strong corporate culture, which is stimulated by various ritual practices. Company philosophies and visions, often as an introduction to long-term plans, are another way of maintaining strong corporate feelings. These help to implement a grass roots decision making process by informal and spontaneous mutual adjustment, called « horizontal coordination ». In a changing environment, this model proves to be more efficient than the traditional hierarchical model, owing to its greater flexibility. In order to stimulate internal communication, various means are used. In Japan, advertising towards potential customers is also a means of internal communication intended to inform and motivate the personnel. So internal and external communication are all one.

\section{AUTEUR}

\section{RENAUD DE MARICOURT}

Renaud de Maricourt. diplômé d'Etudes Politiques de Paris, licencié en droit et en sciences économiques, MBA Northwestern University, est professeur à l'ESCP, où il enseigne le marketing. Il donne aussi un cours sur l'environnement socio-économique des affaires au Japon et sur les méthodes Japonaises de management des entreprises, et à dirigé plusieurs années, à l'ESCP, le programme Concordia destiné à former des jeunes cadres de grandes firmes japonaises à l'environnement des affaired en France. 